\title{
Simulation of Active Compensated Pulsed Alternator with a Laser Flashlamp Load Based on Simplified Model
}

\author{
Pei Yuan*, Kexun Yu*, Caiyong Ye*and Zhang’ao Ren*
}

\begin{abstract}
This paper presents a nontraditional laser power system in which an active compensated pulsed alternator (ACPA) drives a flashlamp directly without the use of capacitor groups. As a result, the volume of the laser system is decreased because of the high energy density of the ACPA. However, the difficulty in matching the output of the alternator with the laser flashlamp is a significant issue and needs to be well analyzed. In order to solve this problem, based on the theory for ACPA, the authors propose a simplified model for the system of ACPA with flashlamp load by the way of circuit simulation. The simulation results preliminarily illuminate how the performance of the ACPA laser power system is affected. Meanwhile, the simulation results can also supply a consultation for future ACPA laser power system design and control.
\end{abstract}

Key words: Active compensated pulsed alternator(ACPA), Flashlamp load, Simulation on simplified model

\section{Introduction}

Traditionally, capacitors, as the energy storage element, are widely used in the power system for laser flashlamps (Fig.1). But the relatively large total volume of the capacitor groups and their complicated charging and discharging circuits extremely limit the movability of the laser system, which leads to inconvenience in real applications [1]. In this case, the replacement of the capacitor groups by a compensated pulsed alternator (CPA) is proposed because of its high energy and power density, as well as having a simpler external circuit [2]. Therefore, the volume of the whole system can be decreased considerably.

However, as a special resistive load, the laser flashlamp requires that the source to deliver tens of kilojoules of energy in hundreds of microseconds $(400 \mu s-600 \mu s)$ [3]. In other words, the pulse width and amplitude of the CPA output current have to meet the requirements of the flashlamp load. According to the characteristics of different compensation forms, which are active compensation, passive compensation and select passive compensation [4], the authors finally chose the active compensated pulsed alternator (ACPA) to drive the laser flashlamp directly. As a result, the size of the whole laser system will be greatly reduced to satisfy the requirements of miniaturization and movability (Fig.2).

* State Key Laboratory of Advanced Electromagnetic Engineering and Technology (AEET), Huazhong University of Science and Technology (HUST), C h i n a .(ypsnow87@sina.com, kexunyu@163.com, cychust2001@163.com,renzando@foxmail.com)

Received 09 October 2012; Accepted 15 November 2012

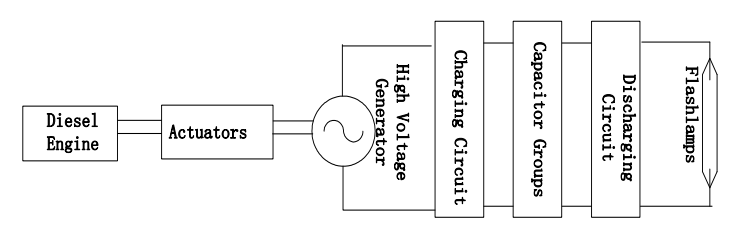

Fig.1. Schematic of a traditional laser power system with capacitors

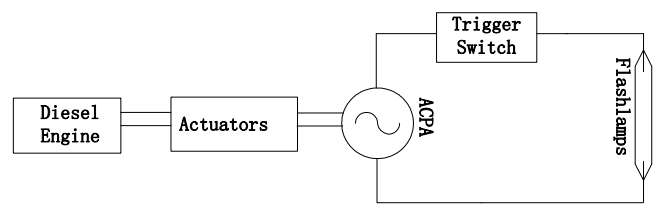

Fig.2. Schematic of ACPA system that directly drives flashlamps

Nevertheless, the problem of matching the parameters between the ACPA and the flashlamp load is complicated. In $[5,6,7]$, the systems are based on the experimental data of the particular compensated pulsed alternators. In $[10,11]$, the authors initially calculated and analyzed the characteristic parameters of the current waveform of the ACPA, as well as the parameters of the machine effects.

The authors will explain theoretically how the ACPA performs with the special resistive load, the flashlamp, which is not discussed in theses references based on the this paper discusses the factors which have an effect on the performance of the ACPA matched with a flashlamp load. 
The model is constructed by the method of circuit simulation, which is different from the methods in the references above.

On this basis, the authors have simulated the performance of the ACPA with the flashlamp load. Meanwhile, some useful conclusions in the references above are also validated from this investigation. The characteristics of the flashlamp load are investigated in this paper and the simulation results can supply a consultation for future ACPA laser power system design and control.

\section{Prinvples and Modeling}

Compared with the ordinary single-phase synchronous generator, the ACPA has an additional coil, named compensating coil, fixed on the same side (stator or rotor) with the field coil. The compensating coil is almost identical to the armature coil. Both of them are connected in series by a brush and slip ring system. In this way, the output power can be increased by flux compression [8] (Fig.3).

The equivalent circuit of ACPA with load is shown in Fig. 4. The ACPA is modeled as an alternating voltage source $e(t)$ in series with a variable internal inductance $L(t)$ and an internal resistance $R_{i} . R_{L}$ represents the resistive flashlamp load. Assuming $e(t)=E_{m} \sin \alpha, E_{m}$ is the no-load induced voltage amplitude. The circuit differential equation can be expressed as below:

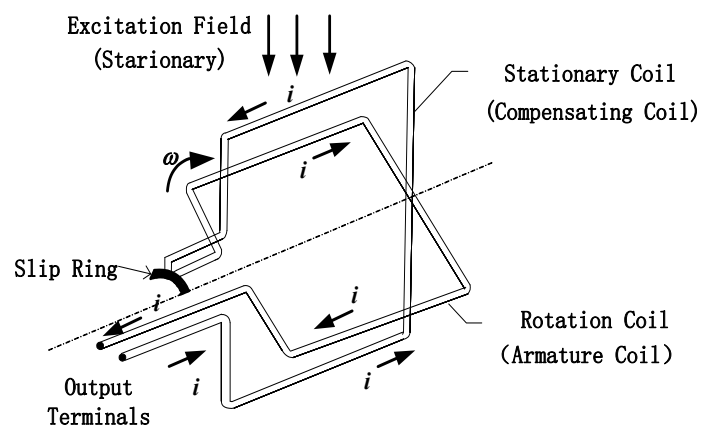

Fig.3. Schematic of ACPA

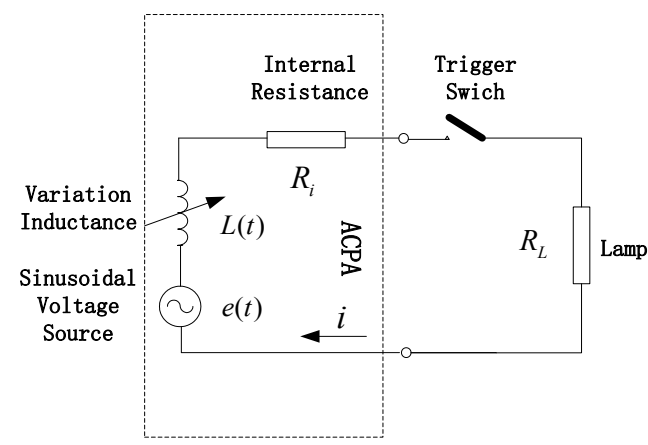

Fig.4. Schematic of the equivalent circuit of ACPA

$$
\frac{d}{d t}[L(t) i]+R i=e(t)
$$

In an ideal machine model, the inductance will vary si nusoidally with the rotor position, from a maximum value $L_{\max }$, to a minimum value $L_{\min }[9]$ :

$$
L(t)=\frac{L_{\max }+L_{\min }}{2}+\frac{L_{\text {max }}-L_{\min }}{2} \cos (\alpha-\delta)
$$

where $\alpha$ is the rotor position angle, more specifically, the electrical angle between the axes of armature winding and field winding. Similarly, $\delta$ is called the compensating angle, which is the electrical angle between the axes of compensating winding and field winding [10].

Then, the compression ratio $\rho$ and the compression factor are defined as follows:

$$
\begin{gathered}
\rho=\frac{L_{\max }}{L_{\min }} \\
\Delta \rho=(\rho-1) /(\rho+1)
\end{gathered}
$$

In terms of the dimensionless quantities (3) and (4), equation (1) may be rewritten.

$$
\begin{gathered}
L(t)=\frac{L_{\min }(1+\rho)}{2}\left[1+\frac{\rho-1}{\rho+1} \cos (\omega t+\theta-\delta)\right] \\
L(t)=\frac{L_{\min }}{1-\Delta \rho}[1+\Delta \rho \cos (\omega t+\theta-\delta)]
\end{gathered}
$$

where $\alpha$ is replaced by $\omega t+\theta, \omega$ is the electrical angular velocity of the ACPA, and $\theta$ is the initial electrical angle between the axes of armature winding and field winding. In other words, $\theta$ is the value of $\alpha$ when trigger time $t$ equals to zero.

Using equations (5) and (6), equation (1) may be expressed as follows:

$$
\begin{aligned}
& -\frac{\Delta \rho L_{\mathrm{mi}}}{1-\Delta \rho} \mathrm{si} \mathrm{nat}+\theta-\delta i j() \\
& +\frac{L_{\mathrm{m} \mathrm{in}}}{1-\Delta \rho}(1+\Delta \rho \cos (\omega t+\theta-\delta)) \frac{d i(t)}{d t} \\
& +R i(t)=e(t)
\end{aligned}
$$

Simulation is done and some equivalent transformations can be done in equation (7):

$$
\begin{aligned}
& u_{1}(t)=-\frac{\Delta \rho L_{\min }}{1-\Delta \rho} \sin (\omega t+\theta-\delta) i(t) \\
& u_{2}(t)=\frac{L_{\min }}{1-\Delta \rho}(1+\Delta \rho \cos (\omega t+\theta-\delta)) \frac{d i(t)}{d t}
\end{aligned}
$$

It can be seen that $u_{1}(t)$ is a current-controlled voltage 
source, while $u_{2}(t)$ is the terminal voltage of a nonlinear inductance which may be simulated by a voltage-controlled current source. The controlling coefficient $\beta$ of $u_{1}(t)$ and the equivalent inductance $L_{e q}$ of $u_{2}(t)$ are shown as follows:

$$
\begin{aligned}
& \beta=-\frac{\Delta \rho L_{\min }}{1-\Delta \rho} \sin (\omega t+\theta-\delta) \\
& \left\{\begin{array}{l}
L_{e q}=\frac{L_{\min }}{1-\Delta \rho}(1+\Delta \rho \cos (\omega t+\theta-\delta)) \\
i(t)=\int \frac{u_{2}(t)}{L_{e q}} d t+i_{0}=\int \frac{u_{2}(t)}{L_{e q}} d t
\end{array}\right.
\end{aligned}
$$

The behavior of the flashlamp may be separated into two phases. They are: (1) the initial streamer breakdown and arc growth to full bore, and (2) the lamp current buildup and decay at full bore. The energy cost of phase (1) is less than $1 \%$ of that of phase (2) [3].That's to say that almost all of theenergy supplied by the ACPA is delivered in phase (2). In other words, the influence of phase(1) is slight.

As a result, phase (1) is ignored and only phase (2) is considered, in order to simplify the model. Under this assumption, the flashlamp can be expressed as a variable resistor [12]. The volt-ampere characteristic equation is written as:

$$
V=K_{0} \sqrt{I}
$$

Where $K_{0}$ is the impedance parameter of a flashlamp. Thus, the simulation model can be constructed with the help of equations (7) to (12).

\section{Analysis of the simulation results}

For the purpose of facilitating the analysis and comparison, the standard per unit form is adopted. The basic value of current $I_{b}$ may be expressed as follows:

$$
I_{b}=\frac{E_{m}}{\omega L_{\min }}
$$

The main idea is that the characteristics of the pulsed current generated by the ACPA, including the waveform, the pulse width and the peak value, are flexibly modulated, in order to match the requirements of the flashlamp load.

Firstly, the ACPA no-load characteristics are discussed And how the output current is influenced by the initial rotor position angle (trigger angle) $\theta$, the compensating angle $\delta$, as well as the compression ratio $\rho$, which are pivotal parameters in ACPA already mentioned above, is explored. The existence of an internal resistor brings about a small decline in the amplitude of the pulsed current. What's more, the internal resistor also leads to a slight asymmetry in the waveform. However, the internal resistor does not change the overall trend of the pulsed current waveform [11]. As a result, it is reasonable to ignore the internal resistor under no-load condition.

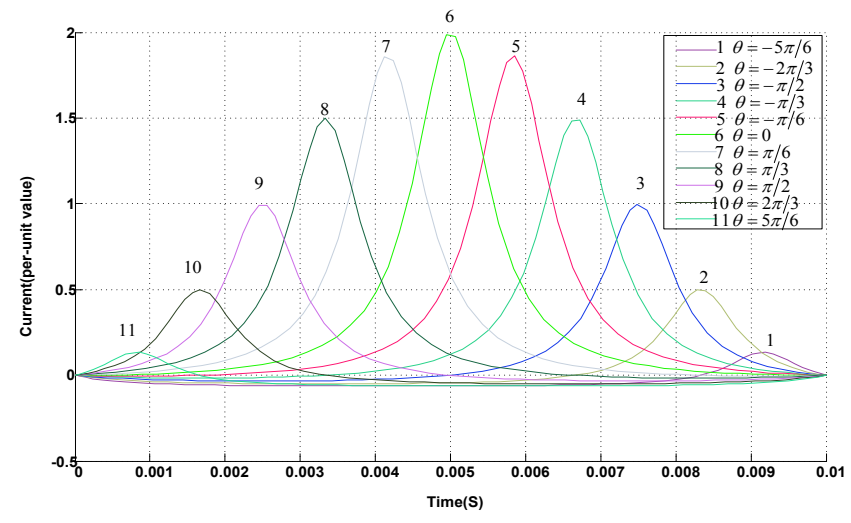

Fig. 5.The variation of the pulsed current waveforms influenced by trigger angle $\theta$

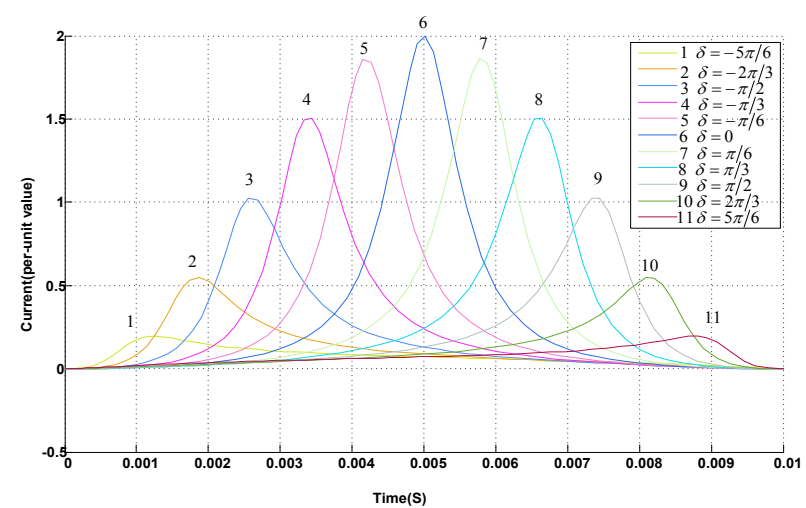

Fig.6. The variation of the pulsed current waveforms influenced by compensating angle $\delta$

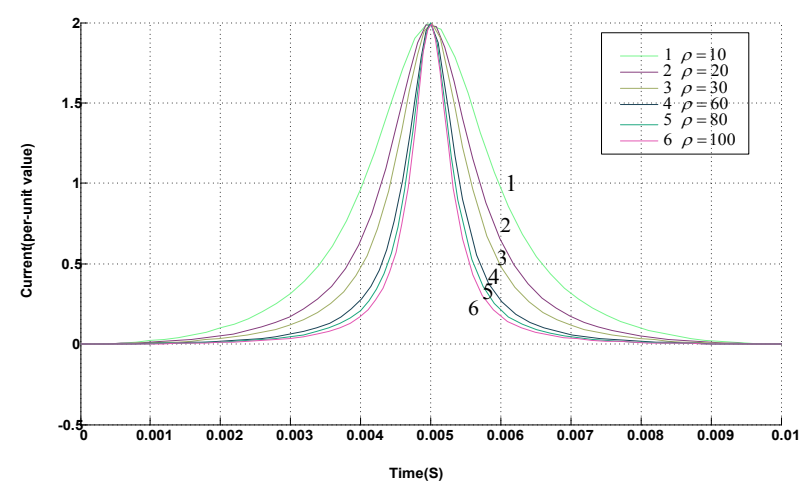

Fig.7. The variation of the pulsed current waveforms influenced by compression ratio $\rho$ 
Secondly, in order to give prominence to the influence of the pivotal parameters above, a constant rotation speed of $n=3000 \mathrm{r} / \mathrm{min}$ and poles $2 P=4$ are assumed. In practical applications, the rotation speed can be varied to meet the different required pulse widths. Also,

$\omega=P \frac{2 \pi n}{60}=2 \times \frac{2 \pi \times 3000}{60}=200 \pi \mathrm{rad} / \mathrm{s}$

Based on the preconditions above, the simulation results are shown as below:

1. The influence of the trigger angle $\theta$.

Assuming $\delta=0$ and $\rho=30$, the values of $\theta$ are varied to obtain the results shown in Fig.5.

It is clear that the peak value of the current decreases with the increase of $|\theta|$, that is to say that the maximum occurs when $\theta$ equals to zero.

2. The influence of the compensating angle $\delta$.

Assuming $\theta=0$ and $\rho=30$, the values of $\delta$ are varied to obtain the results shown in Fig.6.

From Fig.6, we can see that the tendency of the peak value is similar to that for the case of the variation of the trigger angle $\theta$. Identically, the peak value reaches its maximum when $\delta$ equals to zero.

3. The influence of the compression ratio $\rho$.

Assuming $\theta=0$ and $\delta=0$, the values of $\rho$ are varied to obtain the results as shown in Fig.7.

Fig.7 shows that the pulse width of the output current decreases with the increase of $\rho$. That is the larger the compression ratio, the sharper the waveform

The simulation results above validate the theoretical an alysis and formula derivation in [10].

4. The matching between the output of the ACPA and the flashlamp load.

Subsequently, the results above are applied to analyze the ACPA with a flashlamp load. According to the conclusions drawn previously, the trigger angle $\theta$ and the compensating angle $\delta$ are both set to zero so as to acquire the maximum peak value of the output current. The compression ratio $\rho$ is treated as a variable to match the flashlamp loads with different impedance parameters $K_{0}$. The contrastive results are shown in Fig.8, Fig.9, Fig.10 and Fig. 11.

Fig.8 and Fig.9show that an increase of the flashlamp impedance parameter will lead to a decrease of the pulse peak value. Also, the time when the peak value occurs will be advanced.

Compared to Fig.8, Fig.9 doubles the compression ratio (from $\rho=50$ to $\rho=100$ ), but the amplitude of the pulse grows negligibly. Meanwhile, an increase of the ACPA compression ratio will contribute to an increment of the pulse peak value, and the time when the peak value occurs is also delayed, as shown in Fig.10 and Fig.11.

Similarly, Fig.11 drives double the load in contrast to Fig. 10 (from $K_{0}=20 \Omega \square \quad$ to $K_{0}=40 \Omega \square$ '). As a result, the amplitude of the pulse drops noticeably.

Based on the results shown above, the flashlamp load gives more significant impacts on the adaptation while the elevation of the compression ratio has benefit on the driving capacity of the ACPA.

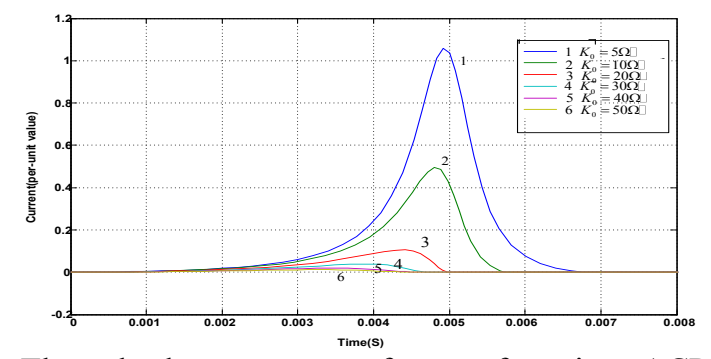

Fig.8. The pulsed current waveforms of a given ACPA with different flashlamps of different impedance parameters $(\rho=50)$

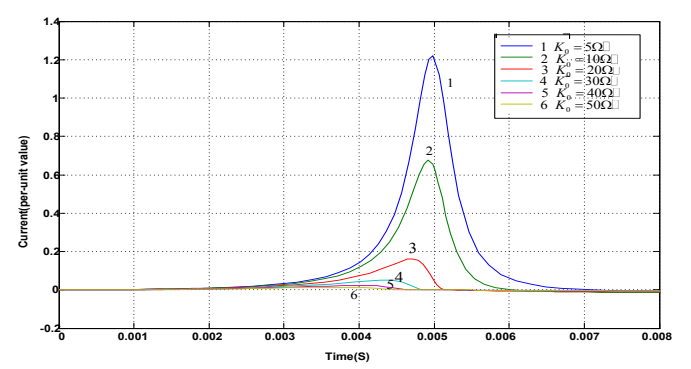

Fig.9. The pulsed current waveforms of a given ACPA with different flashlamps of different impedance parameters $(\rho=100)$

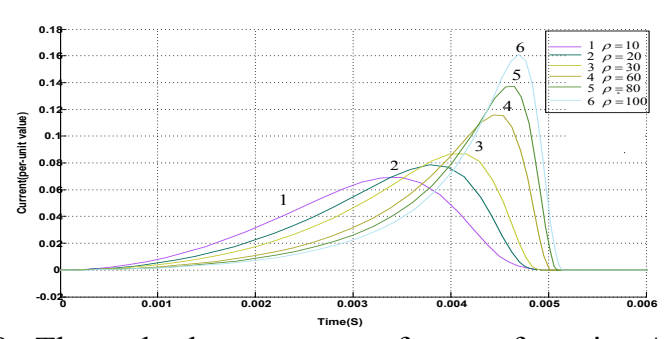

Fig.10. The pulsed current waveforms of varying ACPA compression ratios with a given flashlamp load impedance parameter $\left(K_{0}=20 \Omega \square{ }^{\cdots}\right)$

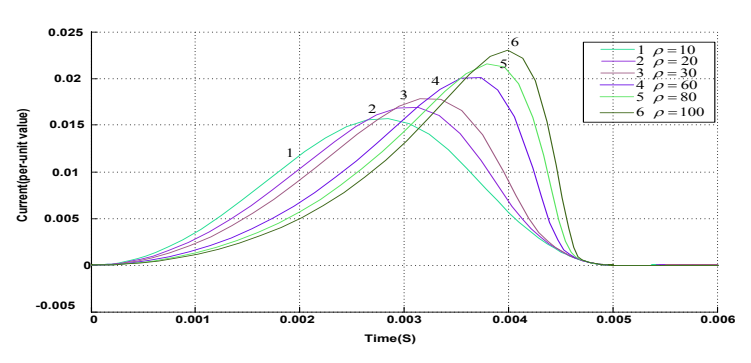


Fig.11. The pulsed current waveforms of varying ACPA compression ratios with a given flashlamp load impedance parameter $\left(K_{0}=40 \Omega \square{ }^{\cdots}\right)$

\section{Conclusion}

Since this nontraditional laser power system has a resistive flashlamp load, it was necessary to consider the impact of the resistance on the ACPA output. Through the analysis above, some conclusions about the matching between the output of the ACPA and the laser flashlamp are given as follows:

1. The trigger angle $\theta$ and the compensating angle $\delta$ both mainly have an effect on the peak value of the output, while the compression ratio $\rho$ greatly affects the pulse width

2. In the actual design and control-of the ACPA, it is beneficial to make the axes of compensating winding and field winding as close as possible to each other in order to achieve a small value of compensating angle $\delta$. What's more, the discharge time may be chosen so that the trigger angle $\theta$ equals to zero. Both of these actions are required to ensure that the maximum peak value is obtained. This conclusion is drawn at with the premise of ignoring the influence of the resistor. The existence of resistive flashlamp load and internal resistor in real applications leads to an acceptable deviation in the results.

3. With an increase in the flashlamp load, the peak value of the ACPA output will decrease and the time when the peak value occurs will be advanced. As a result, corresponding adjustments to the external circuit which is used for controlling the discharge time of the ACPA should be made.

4. The raising of the ACPA compression ratio will make the rising time of the current longer and the falling time shorter, which are both good operating conditions for flashlamps. At the same time, a higher peak value is provided to the flashlamp load. In view of this, the compression ratio may be made as high as possible by reducing the minimum inductance $L_{\min }$.

\section{Acknowledgment}

The author would like to acknowledge the support of the Chinese National Natural Science Foundation (NO. 51077 060).

\section{References}

[1] J. H. Gully, "Power Supply Technology for Electric Guns," IEEE Transactions on Magnetics, vol. 27, no. 1, January, 1991.

[2] Weldon W F, Driga M D, Woodon H H, "Compensated Pulsed Alternator", U. S. Patent No. 4200831, April 29, 1980.

[3] William L. Bird, Hircea D. Driga, David J. T. Mayhall, Michael Brennan, "Pulsed Power Supplies for Laser Flashlamps," Lawrence Livermore Laboratory Subcontract , pp.15-16, October, 1978.

[4] Caiyong Ye, Kexun Yu, Xiaoxu Liu, Yuan Pan, "Investigation on the Current Pulse Formation of Compulsators," High Voltage Engineering, vol. 34, no. 2, February. 2008.

[5] W. L. Bird, D. J. T. Mayhall, W. F. Weldon, H. G. Rylander, and H. H. Woodson, "Applying A Compensated Pulsed Alternator to a Flashlamp Load for Nova - Part II," $2^{\text {nd }}$ IEEE Pulsed Power Conf., Lubbock, TX, USA, June 12-14, 1979.

[6] M. D. Werst, D. E. Perkins, S. B. Pratap, M. L. Spann, and R. F. Thelen, "Testing of a Rapid Fire Compensated Pulsed Alternator System," IEEE Transactions On Magnetics, vol. 25, no. 1, January, 1989.

[7] M. J. Kear, J. W. Gray, J. L. Browning, and D. J. Wilson, "Demonstration Compulsator with Slotted Windings," 6th IEEE Pulsed Power Conf., Arlington, VA, June 29-July 1, 1987.

[8] W. F. Weldon, W. L. Bird, M. D. Driga, K. M. Tolk, H. G. Rylander, H. H. Woodson, "Fundamental Limitations and Design Considerations for Compensated Pulsed Alternators," $2^{\text {nd }}$ IEEE Pulsed Power Conference, 1979.

[9] D. Putley, "Analysis and Modeling Of the Culham Experimental Compulsator," $\sigma^{\text {th }}$ IEEE Pulsed Power Conference, 1987.

[10] Yongqian Xiong, Zhiyun Ma, Libing Zhou, Shizhang Xu, "Effects of Parameters on Current Waveform in Active Compensated Pulsed Alternator," Transactions of China Electrotechnical Society, no. 3, August, 1994.

[11] Yongqiang Xiong, "The Theoretical and Experimental Research on Active Compensated Pulse Alternator [D]," Huazhong University of Science and Technology, pp. 45-48, 1995.

[12] P. A. Winstanley, "The Role of Pulse Power in Flashlamp Pumped Lasers," Pulsed Power '97 (Digest No: 1997/075), IEE Colloquium on IET Conferences, March 19, 4/1-4/3, 1997.

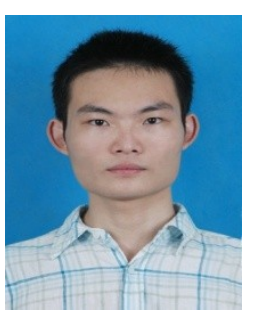

Pei Yuan received the B.S. degree in electrical and electronic engineering from the Huazhong University of Science and Technology (HUST), Wuhan, China, in 2009, where he is currently working toward the Ph.D. degree in electric machines and electric apparatus in the College of Electrical and Electronic Engineering, Huazhong University of Science and Technology (HUST), Wuhan, China. His research areas include electrical machinery and drives and the design of power electronics.

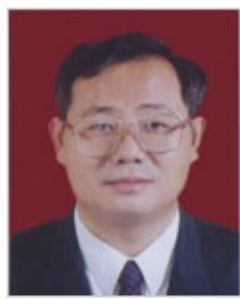

Kexun Yu received the B.S. degree in Shandong University, Ji'nan, China, in 1982. He received the M.S. degree and Ph.D. degree in electrical and electronic engineering in the Huazhong University of Science and Technology (HUST), Wuhan, China, in 1985 and 1989, 
respectively. He is currently with HUST. His research activities are focused on high performance electric drive system, numerical simulation and optimization design of electromagnetic device.

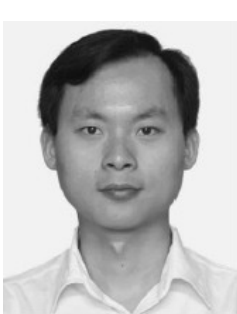

Caiyong Ye received the B.S. degree and Ph.D. degree in electrical and electronic engineering in the Huazhong University of Science and Technology (HUST), Wuhan, China, in 2005 and 2010 , respectively.

$\mathrm{He}$ is currently with HUST. His technology and special electrical machinery.

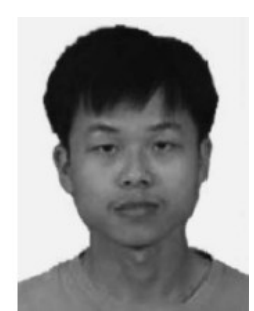

Zhang'ao Ren received the B.S and $\mathrm{Ph}$.D. degree in electrical and electronic engineering from the Huazhong University of Science and Technology, Wuhan, China in 2007 and 2012, respectively. His research area include electrical machinery and drives and the design of power electronics. His recent research activities include dc-pulse charging power supply and transient analysis of HIA. 